\title{
Herramienta educativa para la formación de ingenieros en protecciones eléctricas: relevador de distancia
}

\section{Educational Software Tool for Protection System Engineers. Distance Relay}

\author{
Trujillo-Guajardo L.A. \\ Programa Doctoral en Ingeniería Eléctrica \\ Facultad de Ingeniería Mecánica y Eléctrica \\ Universidad Autónoma de Nuevo León \\ Correo: luistrujillo84@gmail.com
}

\author{
Conde-Enríquez A. \\ Programa Doctoral en Ingeniería Eléctrica \\ Facultad de Ingeniería Mecánica y Eléctrica \\ Universidad Autónoma de Nuevo León \\ Correo:con_de@yahoo.com
}

Información del artículo: recibido: mayo de 2010, aceptado: mayo de 2011

\section{Resumen}

En este artículo se presenta una herramienta computacional gráfica para apoyar la formación de ingenieros en protecciones eléctricas. Los fundamentos teóricos para el diseño de características de operación de relevadores de distancia, así como las rutinas de programación de un relevador de distancia son presentados. La herramienta desarrollada permite la evaluación de las etapas de diseño de relevadores y el análisis de la operación ante eventos reales o simulados. Se presentan algunos casos de ejemplo para ilustrar las actividades didácticas que son posibles de realizar con la herramienta presentada.

\footnotetext{
Abstract

In this article, a graphical software tool is presented; this tool is based on the education of protection system engineers. The theoretical fundaments used for the design of operation characteristics of distance relays and their algorithms are presented. The software allows the evaluation and analysis of real time events or simulated ones of every stage of design of the distance relay. Some example cases are presented to illustrate the activities that could be done with the graphical software tool developed.
}

\section{Descriptores}

- relevador de distancia

- herramienta computacional gráfica

- características de operación

- señales eléctricas de entrada

- filtrado analógico

- filtrado digital

\section{Keywords}

- distance relay

- graphical software tool

- operation characteristics

- electric input signals

- digital filter

- analog filter 


\section{Introducción}

La comprensión del funcionamiento y limitaciones de los sistemas de protección es una parte importante en la formación de ingenieros eléctricos. La enseñanza en clase por parte de académicos es limitada en muchas ocasiones por carecer de elementos demostrativos que permitan evaluar las diferentes condiciones de operación de los sistemas eléctricos de potencia, dificultando la capacidad de transmisión del conocimiento de los sistemas de protección eléctrica.

Por otra parte, las actividades en un laboratorio en muchas ocasiones son limitadas por la falta de recursos para el análisis de los eventos y medios de visualización de las diversas condiciones de operación. Los paquetes profesionales de protecciones además de tener un costo prohibitivo para muchos centros educativos se enfocan en la componente de aplicación más que en la componente didáctica.

Un gran número de instituciones educativas cuentan con recursos limitados para ofrecer un laboratorio de protecciones funcional, las actividades de laboratorio están mayormente enfocadas a la enseñanza de conexión y programación de equipo comercial; sin embargo, el análisis algorítmico y detección de condiciones de mal funcionamiento del relevador son difíciles de reproducir en laboratorio. Las actividades de laboratorio difícilmente pueden cubrir una gama amplia de eventos que permitan mostrar el desempeño de relevadores.

En Brahma et al. (2009), se puntualiza la importancia del diseño de cursos de ingeniería de sistemas de potencia con una urgente necesidad de identificar y desarrollar los materiales necesarios para la educación y la formación de los ingenieros de la protección futura, este trabajo está orientado en esa dirección.

En la búsqueda de materiales que ofrezcan recursos didácticos adecuados se han creado laboratorios para ofrecer una componente de enseñanza del sistema eléctrico de potencia. En Wei et al. (2002) se describe la conformación de un laboratorio de simulación de sistemas de potencia a escala de un sistema trifásico para mejorar las actividades de investigación y enseñanza de protecciones; se reproducen las condiciones de operación estáticas y dinámicas de relevadores de protección . En Huang et al. (1991) se presenta un recurso gráfico para el análisis y diseño de sistemas de potencia, el cual provee de herramientas para facilitar la creación y modificación de aplicaciones de análisis de sistemas de potencia, la aplicación permite la comunicación con programas externos para la simulación de sistemas de potencia.
En Jian (1998) se describe un software interactivo PowerGraf para el apoyo del proceso de aprendizaje en clase, laboratorio y auto-aprendizaje. La herramienta gráfica permite la visualización de resultados en forma directa con el unifilar de la red, además permite la interacción con rutinas elaboradas en Matlab. Un complemento a estas actividades es el desarrollo de recursos gráficos con el objeto de visualización de fenómenos que pueden provenir de eventos generados en un laboratorio físico, o de un software comercial para el modelado y análisis de sistemas eléctricos de potencia. En Peterson et al. (1991) se incorporan rutinas de relevadores de protección en forma modular en EMTP (Electromagnetic Transient Program, programa de transitorios electromagnéticos) y se presentan los resultados del modelo de un relevador direccional de sobrecorriente, este modelo tiene el objetivo de mejorar los procedimientos de educación, aplicación y diseño de relevadores. En McLaren et al. (2001) se presenta una revisión de los modelos utilizados para relevadores de protección, su validación y aplicaciones futuras en programas de transitorios, y su simulación en estado dinámico en tiempo real en la operación de la red eléctrica.

La directriz en los recursos didácticos para el aprendizaje de sistemas de potencia y particularmente en protecciones, ha sido aportar recursos gráficos (Huang et al., 2002; Jian et al., 1998; Jordán et al., 1997; Hirsch, 1997; Salon, 1983; Wachal et al., 1984; Alvarado et al., 1984, Lo et al., 1986; Papadopoulos et al., 1987, Alvarado et al., 1987, Daneshdoost et al., 1989; Yu et al., 1989), esto se debe a que la visualización de la operación de las protecciones se realiza por medio de la comparación de condiciones de operación determinadas en una característica de operación del relevador. Mediante esta comparación se evalúa y determinan las condiciones de operación particulares que pueden comprometer la operación de la protección. Debido al incremento y factibilidad de comunicaciones en los sistemas eléctricos, la protección pasa de ser un dispositivo de control independiente del sistema a un elemento integral de la operación de la red; la evaluación dinámica de la operación de la protección debe ser simulada en conjunto con el sistema eléctrico.

Los relevadores de distancia son un componente del esquema de protección de redes eléctricas que presenta una mayor complejidad algorítmica, facilitando por consiguiente su aplicación y proceso de ajuste. Sin embargo, es sujeto a diferentes fuentes de error (Cook, 1985; Warrington et al., 1969), el análisis del comportamiento del relevador ante dichos escenarios es cubierto en forma teórica de manera adecuada; sin embargo, emular tales escenarios en laboratorio es deseable y 
aconsejable para la formación de futuros ingenieros en protecciones (Brahma et al., 2009), este aspecto puede ser cubierto de manera satisfactoria con una herramienta computacional que permita emular diferentes escenarios y la visualización de la operación del relevador en el plano complejo con la trayectoria de impedancia y su característica de operación.

La herramienta computacional presentada para el análisis del diseño y operación de relevadores de distancia ofrece una componente didáctica, que permite extender las actividades de enseñanza en la protección de sistemas eléctricos. Es posible la realización de diversas actividades didácticas, tal como la conformación y diseño de las características de operación de relevadores de distancia, disponiendo de controles que permitan configurar características de operación comúnmente usadas tal como impedancia, mho, mho desplazado y cuadrilateral. El análisis de eventos se realiza mediante la lectura de vectores columna de datos de eventos reales o simulados en cualquier plataforma gráfica comercial o académica. Los eventos simulados pueden ser generados mediante el apoyo de simuladores digitales tales como: EMTP, PSCAD, MATLAB, ETAP, NEPLAN, PSS y otros. Mediante la generación de las señales en vectores de datos es posible reproducir una gama amplia de situaciones operativas y explorar con mayor resolución diversas condiciones operativas de los relevadores. Por lo anterior, se puede evaluar la operación del relevador en condiciones de cargabilidad, fallas sólidas o a través de alta impedancia, oscilaciones de potencia, presencia de dispositivos FACTS o compensación serie, etcétera.

El análisis de la operación del relevador de distancia en todas sus zonas de protección y el análisis de esquemas de bloqueo o disparo por oscilaciones de potencia no son considerados en el trabajo presentado; sin embargo, estas actividades pueden realizarse de forma individual, la detección de la operación por zona ante una falla y la visualización de la trayectoria de oscilación son posibles. No obstante, la herramienta computacional propuesta permite simplificar y profundizar en la enseñanza de protecciones de alumnos de pregrado y postgrado en un espectro amplio de tópicos y escenarios dinámicos de operación de la protección.

\section{Estructura funcional}

La evaluación del desempeño de un relevador de distancia está en función de su capacidad de detectar fallas dentro de su zona de protección. El relevador de distancia opera cuando la impedancia medida es menor a su ajuste, en esta condición, la impedancia del sistema pe- netra en la característica de operación, la decisión de disparo debe ser considerada, debido a que en el sistema están presentes fenómenos que pueden provocar penetración de la característica sin ser una condición de falla, tal como las oscilaciones de potencia. El algoritmo del relevador debe recibir los fasores de voltaje y corriente para determinar la condición de operación (McLaren et al., 2001; Cook, 1985; Warrington et al., 1969) estas señales de entrada deben ser acondicionadas (figura 1).

Las señales eléctricas de entrada al relevador de protección provenientes del secundario de los transformadores de corriente y potencial están compuestas de señales, que si bien son representativas del fenómeno eléctrico, no son requeridas para determinar el criterio de operación del relevador, debido a que presentan un carácter aleatorio que imposibilita la determinación de ubicación de la falla; el procesamiento digital debe eliminar las cantidades no deseadas y retener las cantidades de interés. Tanto en relevadores de distancia como de sobrecorriente, el filtrado de la señal debe eliminar las componentes de corriente directa, los transitorios de transformadores de instrumentos, reflexiones de onda viajera y otras interferencias (Cook, 1985). La operación del relevador debe establecerse únicamente con la componente fundamental a la frecuencia nominal, debido a que esta componente de la señal es afectada proporcionalmente por la ubicación de la falla.

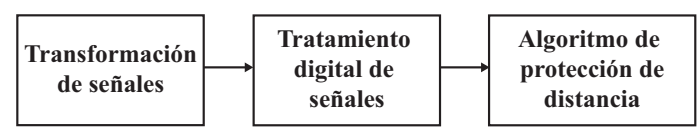

Figura 1. Acondicionamiento de señales eléctricas de entrada

El filtrado de la señal de entrada impacta directamente el desempeño del algoritmo del relevador, afectando la exactitud en la operación dependiendo del rechazo de componentes no deseadas e incrementando la carga de procesamiento. El tiempo total de operación del relevador depende del proceso de filtrado analógico y/o digital seleccionado.

La descripción de las etapas de estructura funcional de un relevador de distancia se presenta desde un enfoque didáctico como justificación de la herramienta computacional presentada.

\section{Transformación de señales}

La saturación de los Transformadores de Corriente (TC) (IEEE Standard, 1993; Tziouvaras et al., 2000) aún cuando impacta el desempeño de los relevadores de distancia, generalmente está presente en condiciones 
muy específicas de operación y de falla, estando directamente asociada al dimensionamiento del transformador, ya que su efecto puede reducirse significativamente. La aplicación de CCVT (Coupling Capacitor Voltage Transformer, transformador de voltaje de acoplamiento capacitivo), (Tziouvaras et al., 2000) para la medición de voltaje, imprime un transitorio en la señal de voltaje medida por el relevador. Debido a que la naturaleza del transitorio depende del instante de la falla, generalmente no se compensa y su mitigación se logra mediante un tiempo de retardo en el relevador (Westinghouse Electric Corpotarion, 1976). La no linealidad de la respuesta de los transformadores de instrumentos no está simulada en la herramienta propuesta, es decir, no se disponen de los modelos matemáticos de los transformadores, el efecto de la saturación de los transformadores en los relevadores de distancia puede analizarse con la herramienta propuesta, introduciendo señales saturadas generadas en algún paquete de simulación académico o comercial.

\section{Tratamiento digital de señales}

Es necesario incorporar dos etapas de filtrado para eliminar las componentes frecuenciales indeseadas, como ruido, armónicas y componentes de corriente directa. Ya que la magnitud o polaridad no son representativas de la ubicación de la falla, se consideran fuentes de ruido que pueden afectar la selectividad del relevador (figura 2).

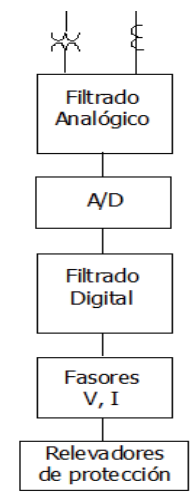

Figura 2. Estructura para el procesamiento de las señales para propósitos de protección

Se dispone de dos etapas de filtrado analógico y digital con el objeto de tener la configuración que presente menor retardo. Generalmente, el filtro analógico utilizado es el filtro Butterworth de $2^{\circ}$ o $4^{\circ}$ orden, con una frecuencia de corte de $360 \mathrm{~Hz}$ (figura 3), se prefiere este filtro por tener respuestas planas en la banda de paso y mo- no-tónica decreciente en la banda de parada (Proakis et al., 1998).

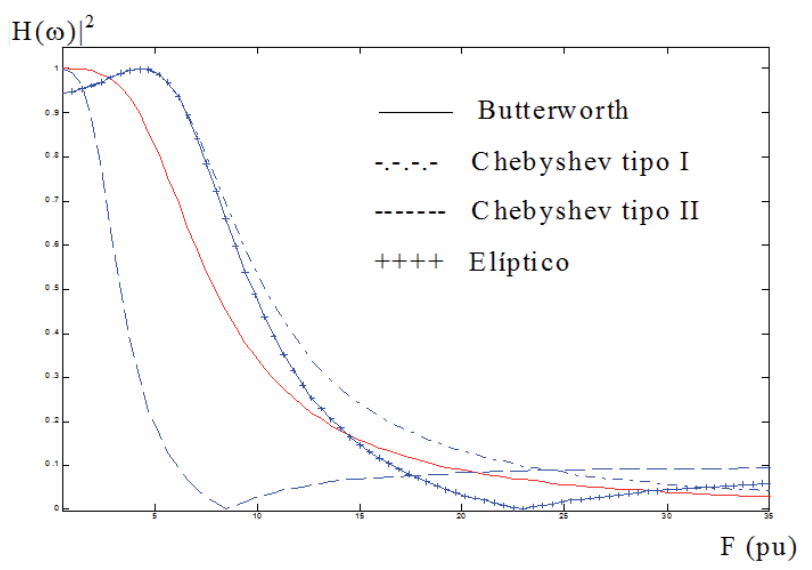

Figura 3. Respuestas a la frecuencia de filtros analógicos

Posterior a la etapa de filtrado analógico se realiza el proceso de digitalización de la señal analógica, el incremento en la frecuencia de muestreo permite lograr un incremento sustancial en la resolución de la señal, pero incrementa la carga del procesador. La reducción del efecto de "aliasing", se obtiene mediante una sintonización entre el filtrado analógico y el filtrado digital, permitiendo un traslape de las frecuencias de filtrado es posible eliminar el filtrado analógico mediante el sobremuestreo de la señal.

El filtrado digital se realiza con filtros tipo FIR (Finite Impulse Response, respuesta impulsional finita), ya que no hay recursión, es decir, la salida depende sólo de la entrada y no de valores pasados de la salida, la memoria de las condiciones previas de la señal no tiene beneficio en la determinación de la condición de falla por el relevador. Además, los filtros IIR (Infinite Impulse Response, respuesta impulsional infinita) producen en general distorsión de fase, contrario a los FIR que son de fase lineal. Esta condición permite que su respuesta a la frecuencia tenga ceros naturales en las frecuencias armónicas, permitiendo un rechazo de estas componentes (figura 4). Se utiliza regularmente el filtro tipo Fourier o coseno.

Teniendo los fasores de las señales de voltaje y corriente de la frecuencia fundamental, se puede representar la impedancia aparente en un plano complejo para efectos de visualización en la operación del relevador, tal como se observa en la figura 5. Sin embargo, la determinación de la condición de disparo no se efectúa mediante la comparación de impedancias, el proceso de toma de decisión de disparo se describe en la siguiente sección. 


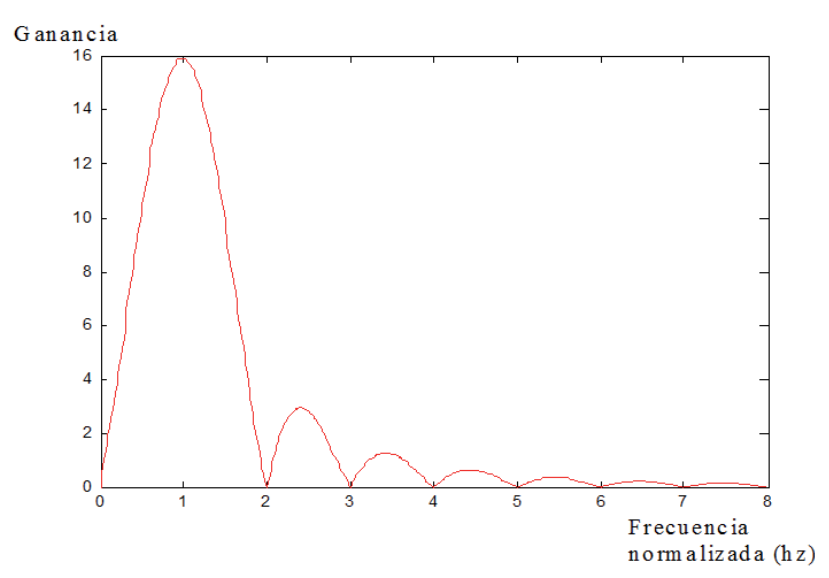

Figura 4. Respuesta a la frecuencia de un filtro de respuesta impulsiva finita (FIR)

\section{Algoritmo del relevador de distancia}

El relevador origina una señal discreta de salida que depende del cumplimiento de determinadas condiciones por las señales eléctricas de entrada. La condición de operación se determina mediante la comparación de señales. Esta comparación puede ser comparación de amplitud o comparación de fase (Cook, 1985).

Las señales de entrada a los comparadores dependen linealmente de las señales de entrada al órgano de medición $\overline{V_{r}}$ e $\overline{I_{r}}$ de acuerdo con las expresiones siguientes:

$$
\begin{aligned}
& S_{0} \dot{o} S_{1}=\bar{K}_{1} \cdot \bar{V}_{r}+\bar{Z}_{R 1} \cdot \bar{I}_{r} \\
& S_{R} \dot{o} S_{2}=\bar{K}_{2} \cdot \bar{V}_{r}+\bar{Z}_{R 2} \cdot \bar{I}_{r}
\end{aligned}
$$

donde $\bar{K}_{1}, \bar{K}_{2}, \bar{Z}_{R 1}, \bar{Z}_{R 2}$ son coeficientes constantes complejos, $S_{0}$ y $S_{R}$ representan señales de entrada de operación y polarización, respectivamente para un comparador de amplitud, $S_{1}$ y $S_{2}$ similarmente para un comparador de fase. Tratando de mejorar la selectividad y funcionalidad de la protección de distancia, históricamente se han desarrollado características de operación diversas, esta diversidad de características de operación se obtiene mediante la variación de los coeficientes de las ecuaciones en (1).

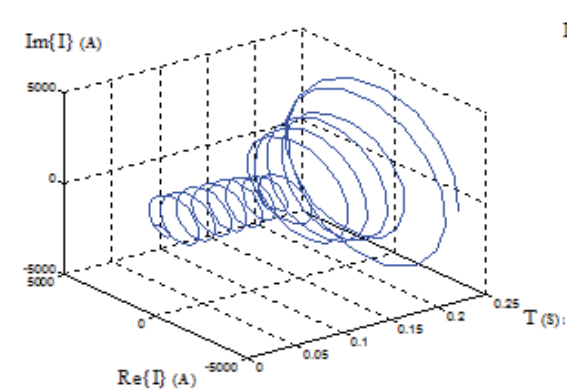

(a)

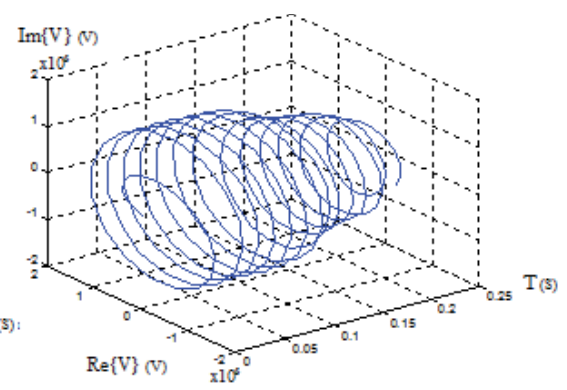

(b)

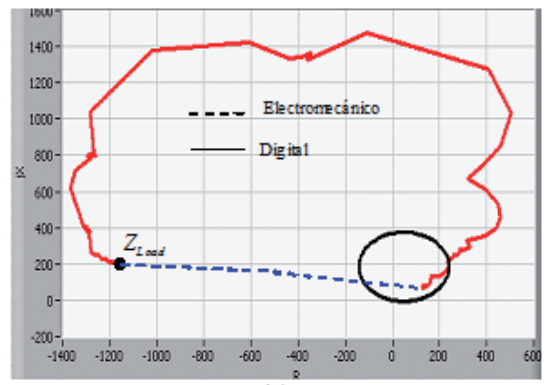

(c)
Figura 5. Representación de la impedancia aparente en un relevador digital de distancia, a) fasor de corriente, b) fasor de voltaje, c) impedancia aparente en el plano complejo 
Las señales de (1) tienen implícitamente una referencia direccional, la señal de operación depende de la dirección de corriente, entonces la señal de referencia debe suplirse por $S_{2}$ o $S_{R^{\prime}}$ la cual es la cantidad de polarización. Por esta razón, para la conformación de las diferentes características de operación direccionales de relevadores de distancia, las señales $S_{2}$ o $S_{R}$ no dependen de la corriente, en cambio, las características impedancia o mho desplazado, que no son direccionales o tienen una direccional controlada, si presentan el término de corriente. Estas afirmaciones se observan en la tabla 1.

En los comparadores se evalúa la amplitud o fase de las señales de entrada, esto representa una rectificación de las señales analógicas y una transformación en valores representativos de voltaje, se observa que los factores de las corrientes en (1) siempre son impedancias.

A esta impedancia se le conoce como impedancia réplica que representa la impedancia de la línea para la señal de operación, y en la señal de polarización, la impedancia representará el desplazamiento de la característica fuera del origen (mho desplazada).

Las señales de comparación son una sumatoria de voltajes, debido a la herencia de relevadores analógicos; sin embargo, también pueden ser corrientes. En la figura 6 se muestran los comparadores de amplitud, fase y sus condiciones de operación.

Los ángulos $\beta_{1}$ y $\beta_{2}$, llamados ángulos coincidentes, establecen el límite angular para la comparación de fase, estos valores son definidos para cada característica de operación, en cambio el ángulo $\beta$ se define por las señales de comparación: $\beta=$ argumento $\left(S_{1} / S_{2}\right)$.

En la figura 7 se presenta un ejemplo de visualización de la característica de un relevador de distancia; la variación de la forma de la característica se obtiene mediante la variación de los ángulos coincidentes.
La estructura de las señales de comparación es más simple para el comparador de fase (tabla 1), por tal motivo, éste es el esquema de comparación utilizado para el diseño de relevadores de distancia. (a)

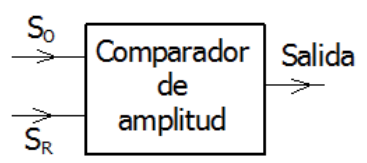

$\left|S_{R}\right| \leq\left|S_{0}\right|$ (b)

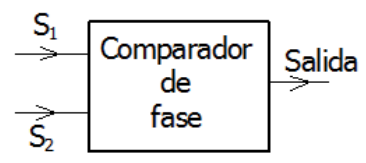

$-\beta_{1} \leq \beta \leq \beta_{2}$
Figura 6. Comparadores de relevadores de distancia

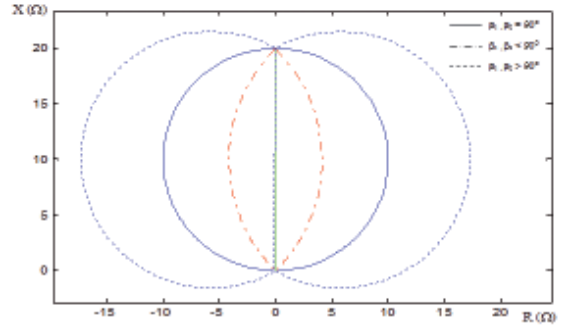

(a)

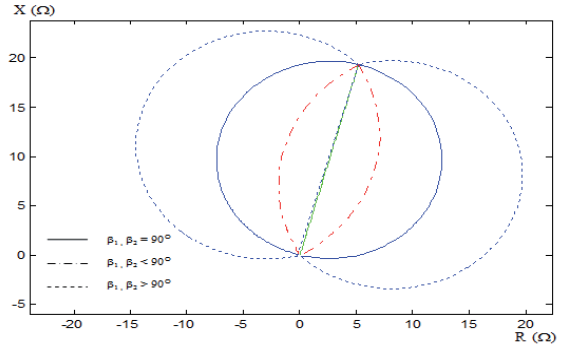

(b)

Figura 7. Variación de los ángulos coincidentes de una característica mho, a) ángulo de sensibilidad $\theta_{1}=90$, b) ángulo de sensibilidad $\theta_{1}=75$

\begin{tabular}{lcccc}
\hline & \multicolumn{2}{c}{ Comparación de fase } & \multicolumn{2}{c}{ Comparación de Amplitud } \\
\hline Tipo de relevador & $\bar{E}_{1}$ & $\bar{E}_{2}$ & $\bar{E}_{1}$ & $\bar{E}_{2}$ \\
(Operación) & (Polarización) & (Operación) & (Polarización) \\
Direccional & $\bar{Z}_{a r \max } \bar{I}_{r}$ & $\bar{V}_{r}$ & $\bar{Z}_{a r \max } \overline{\bar{X}}_{r}-\bar{V}_{r}$ & $\bar{Z}_{a r \max } \bar{I}_{r}+\bar{V}_{r}$ \\
Impedancia & $\bar{Z}_{a r} \bar{I}_{r}-\bar{V}_{r}$ & $\bar{Z}_{a r} \bar{I}_{r}+\bar{V}_{r}$ & $\bar{Z}_{a r} \bar{I}_{r}$ & $\bar{V}_{r}$ \\
Mho & $\bar{Z}_{a r \max } \bar{I}_{r}-\bar{V}_{r}$ & $\bar{V}_{r}$ & $\bar{Z}_{a r \max } \bar{I}_{r}$ & $2 \bar{V}_{r}-\bar{Z}_{a r \max } \bar{I}_{r}$ \\
Mho Desplazado & $\bar{Z}_{a r \max } \bar{I}_{r}-\bar{Z}_{d} \bar{I}_{r}$ & $\bar{V}_{r}-\bar{Z}_{d} \bar{I}_{r}$ & $\bar{Z}_{a r \max } \bar{I}_{r}-\bar{Z}_{d} \bar{I}_{r}$ & $2 \bar{V}_{r-} \bar{Z}_{a r \max } \bar{I}_{r}-\bar{Z}_{d} \bar{I}_{r}$ \\
Impedancia ángulo & $\bar{Z}_{a r \min } \bar{I}_{r}-\bar{V}_{r}$ & $\bar{Z}_{a r \min } \bar{I}_{r}$ & $2 \bar{Z}_{a r \min } \bar{I}_{r}-\bar{V}_{r}$ & $\bar{V}_{r}$ \\
\hline
\end{tabular}

Tabla 1. Señales de comparación 
El algoritmo de programación del relevador de distancia se representa mediante el diagrama de flujo de la figura 8. Las etapas de tratamiento digital de las señales de entrada fueron descritas en la sección de tratamiento digital de señales.

Determinados los fasores correspondientes a la componente fundamental del voltaje y la corriente, se forman las señales de comparación en función de la característica deseada, la condición de operación es establecida según lo descrito en la figura 6 . La verificación de la condición de operación, así como la formación de las señales $S_{1}$ y $S_{2}$, deberá realizarse para cada periodo de muestreo, es decir, para cada nueva muestra de la señal se forma la ventana de datos descartando la última muestra de la ventana, se determinan nuevos fasores y se evalúa la operación del relevador. Muchos fabricantes utilizan arrancadores del algoritmo para evitar un procesamiento continuo y sobrecarga del procesador (Gerhard, 1999).

Por ejemplo, estos arrancadores pueden ser detectores de falla de sobrecorriente. La decisión de disparo debe evaluarse; si esta condición se sostiene durante cierto número de muestras el contacto del relevador se cierra, energizando el circuito de disparo del interruptor de potencia. Con esto se evita la operación en falso del relevador ante oscilaciones de potencia que entran y salen de la característica o fallas en otras zonas de operación con penetración temporal.

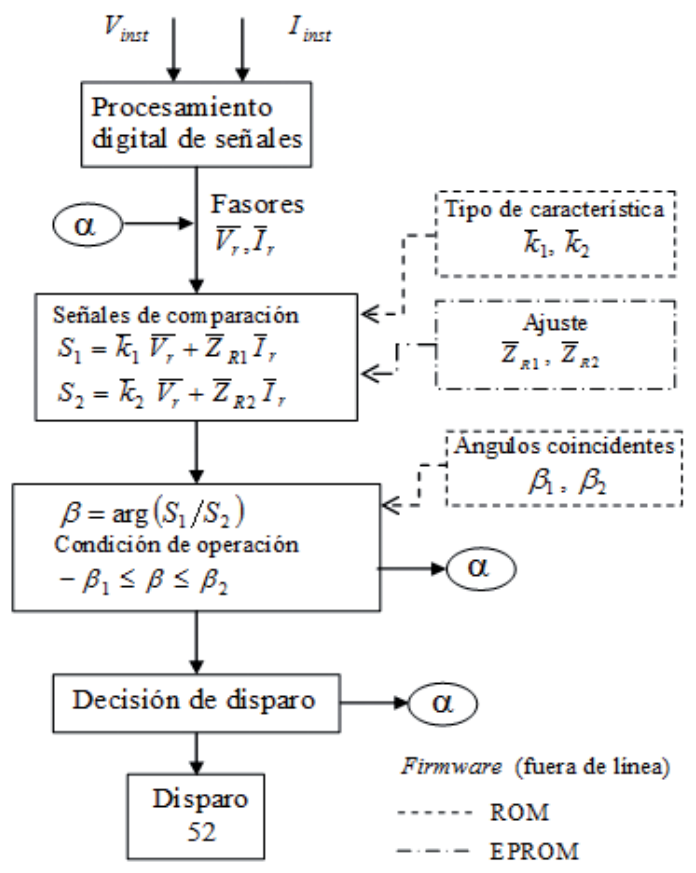

Figura 8. Algoritmo de un relevador de distancia con comparador de fase

\section{Implementación algorítmica en un ambiente de programación gráfico}

El programa general del relevador virtual fue programado en Labview (1997), su estructura funcional se muestra en el diagrama de la figura 9. Se conforman dos modos de prueba, uno para el diseño de la característica de operación y otro para el modo de evaluación mediante la lectura de archivos externos. En el modo de diseño se presentan las alternativas que permiten definir diferentes características de operación, la conformación y diseño de estas características se visualizan en el plano complejo impedancia. El modo de prueba permite extraer datos de archivos externos en formato ASCII generados en programas de simulación (como el EMTP, PSCAD, Matlab, etcétera), o de archivos de datos de registros de fallas reales para la reproducción y validación de la operación del relevador. La herramienta ofrece la versatilidad para la variación de parámetros de ajuste, filtrado analógico y digital. Posterior a la lectura de las señales se realiza el procesamiento digital de la señal tal como se describió en la sección anterior. En el algoritmo del relevador de distancia se define el tipo de característica y se realiza la detección de la condición de operación emitiendo la orden de disparo.

La subrutina de señalización realiza la emisión de la señal de disparo mediante la activación de controles luminosos en el panel frontal una vez cumplida la condición de operación y el tiempo de decisión de disparo. Esto se visualiza en el plano complejo impedancia.

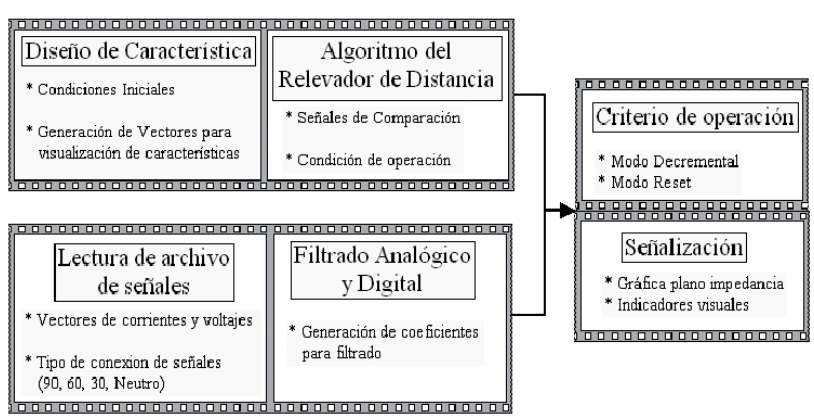

Figura 9. Estructura lógica de la implementación del relevador de distancia en una herramienta de programación gráfica

\section{Actividades didácticas}

\section{Diseño de características de operación}

En este modo de operación se define la característica de operación del relevador, se incluyen las características tipo impedancia, Mho, Mho-desplazada y Cuadrilateral (figura 10). En forma gráfica se indican las variables 
involucradas en el diseño, los comparadores de fase y amplitud están incluidos. La característica de operación se muestra en el plano complejo impedancia.

En la figura 10 se presentan las características de operación obtenidas y los parámetros de ajuste que fue-

(a)

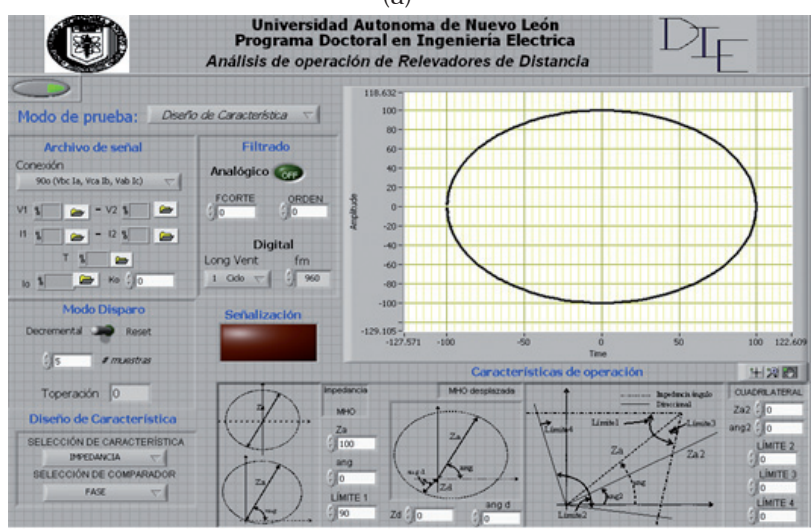

(c)

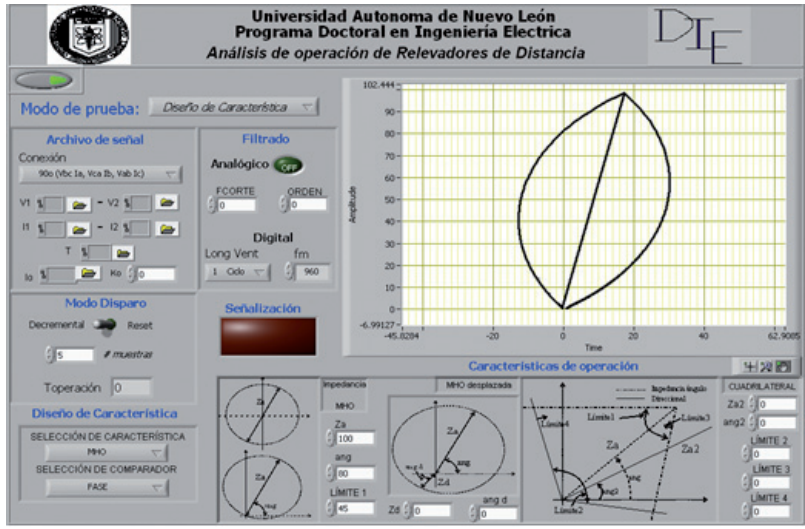

(e)

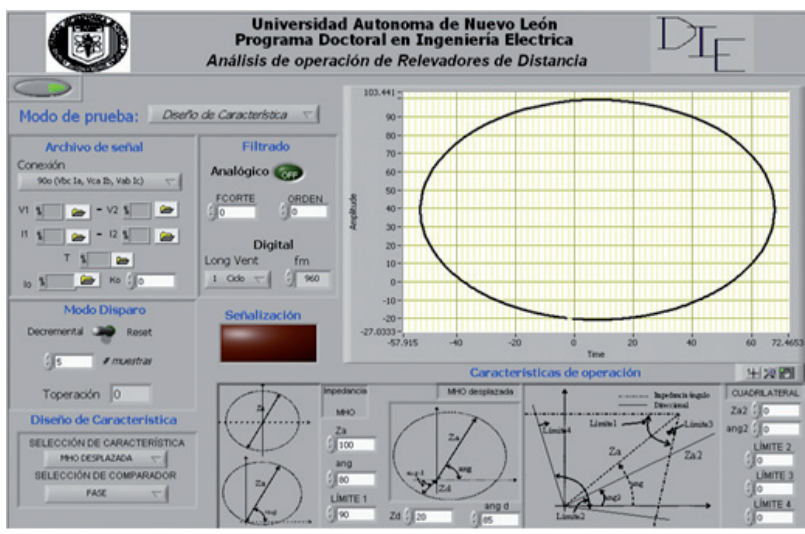

ron definidos. En el caso del diseño de la característica cuadrilateral, con la finalidad de igualar la versión de diseño de algunos relevadores comerciales se disponen cuatro comparadores. Con esto es posible tener cuatro grados de libertad para el diseño de la característica.

(b)

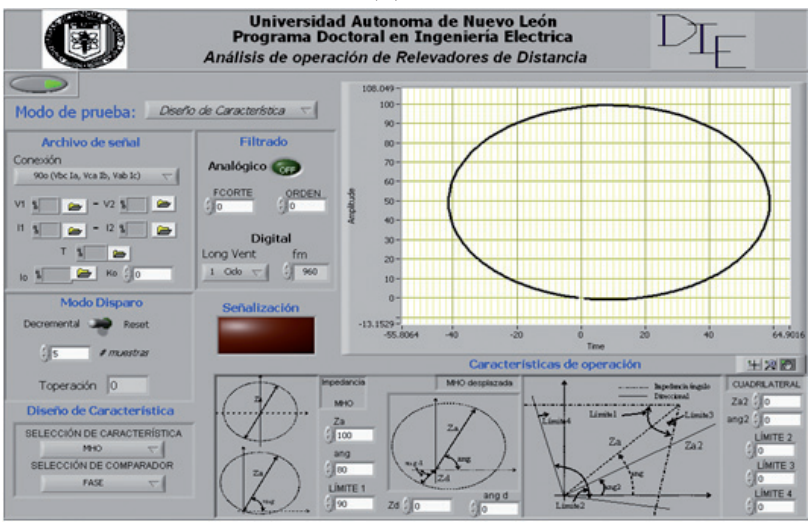

(d)

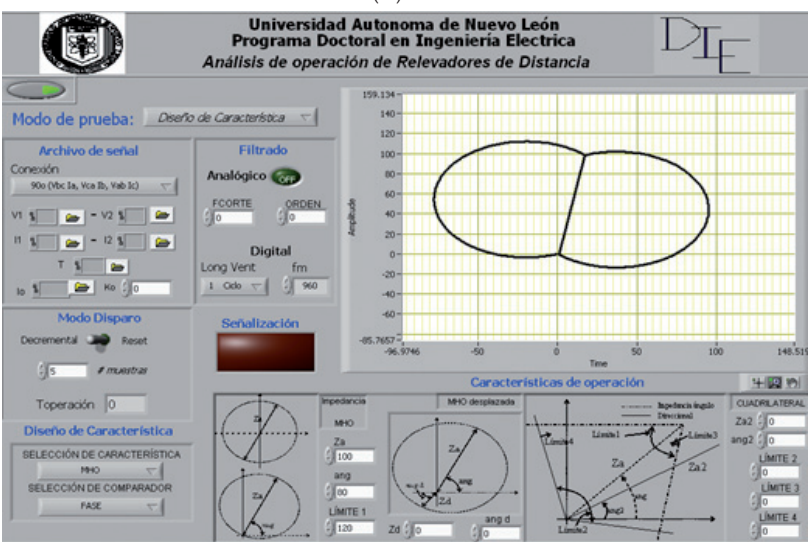

(f)

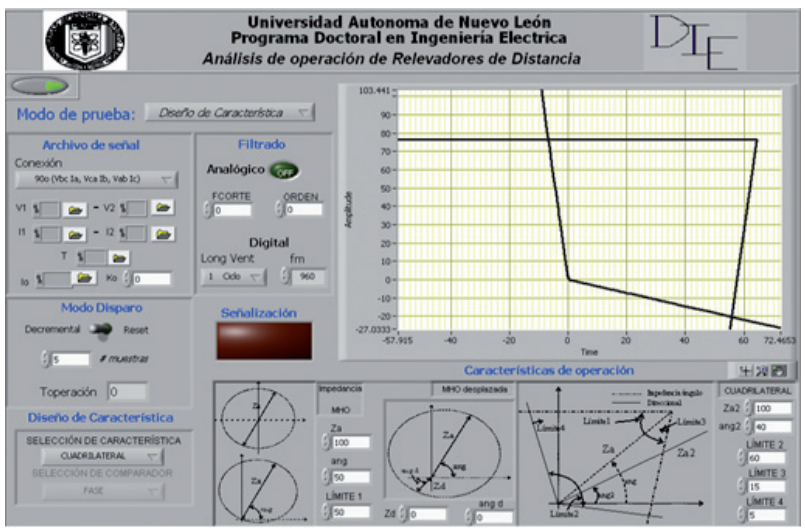

Figura 10. Diseño de características de operación en la herramienta gráfica, a) impedancia, b) Mho, c) lente, d) tomate, e) Mho desplazada, f) cuadrilateral 


\section{Validación de la operación de relevadores}

El análisis de eventos se puede realizar mediante el registro de eventos reales o simulados. En este trabajo se utilizó una señal de prueba de un registro oscilográfico de un evento real en la red de $230 \mathrm{kV}$ en el sistema interconectado nacional y el registro de operación de un relevador de distancia. La prueba tiene como objetivo reproducir la operación del relevador en campo a fin de validar su operación. Adicionalmente, por medio de la herramienta es posible la visualización de la cargabilidad de la red, así como la evaluación del ajuste y tipo de característica.

El registro corresponde a una falla monofásica en la fase $a$ en línea de $230 \mathrm{kV}$ con una impedancia serie de $480 \Omega$. El relevador de campo tiene una característica de operación tipo mho con una impedancia de arranque de valor $382<75^{\circ} \Omega$ ( $80 \%$ de la impedancia de línea). El registro del evento se muestra en la figura 11 utilizando una aplicación comercial.

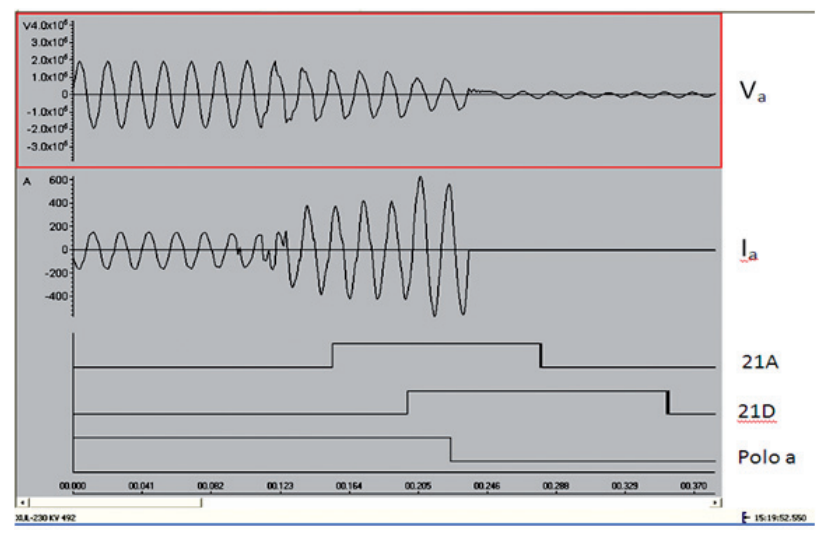

Figura 11. Registro de evento real

Comúnmente la visualización de la operación de relevadores se realiza por medio del registro del tiempo del accionamiento de contactos. En el esquema de protección utilizado en la línea fallada se dispone de un relevador electromecánico y un relevador digital como protección de doble primario, se observa que el tiempo de operación del relevador electromecánico es menor al digital, esto debido al retardo involucrado para el procesamiento de la señal (efecto de ventaneo) como se describió en la sección de estructura funcional. Como se puede observar en el registro del evento (figura 11), la secuencia de operación está dada por la detección de la falla en el relevador analógico, 1 1 1 2 ciclo después de que ésta se presenta. El relevador digital realiza la detección $4 \frac{1}{2} 2$ ciclos después de presentarse la falla y 3 ciclos después de que el relevador analógico la detectara, la aper- tura del polo $a$ se realiza 6 1/2 después de ocurrida la falla.

Del resultado obtenido (figura 12), se pueden mencionar las siguientes observaciones:

- El tiempo de operación del relevador virtual fue similar al relevador del evento real (21D). El tiempo de operación mostrado en la aplicación corresponde desde el inicio de la señal hasta la salida de disparo del relevador.

- Al igual que en el registro del evento real, el relevador virtual operó observando el evento atrás, debido a que se invirtió el flujo de potencia por el tipo de falla.

- La trayectoria que presenta la impedancia de falla no es una línea recta por el filtrado que se realiza sobre las señales eléctricas de entrada, esto aumenta el tiempo de operación en el relevador digital, lo cual se puede observar también en el registro del evento.

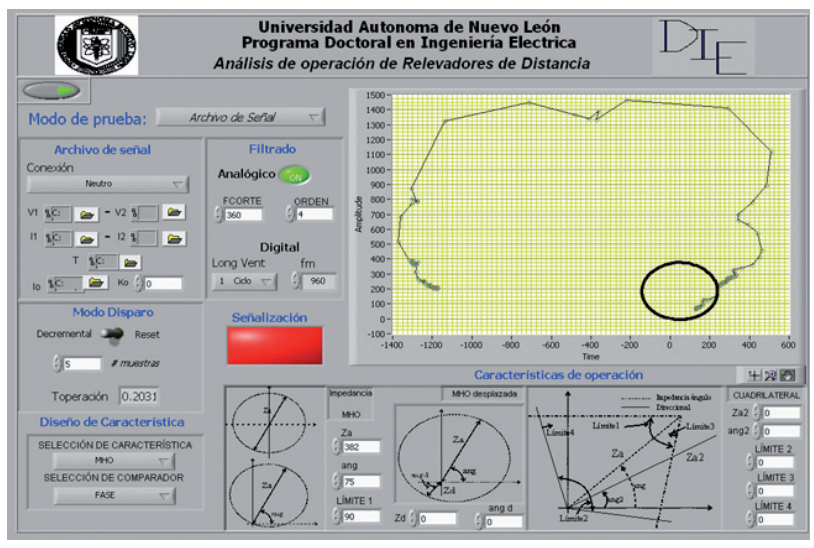

Figura 12. Reconstrucción de la operación de un relevador de distancia en un evento real

\section{Simulación de falla en la aplicación comercial}

En el software de simulación PSCAD se realizó la simulación de una falla y de una oscilación de potencia. Los eventos fueron almacenados en archivos de texto, se realizó el diezmado de la señal y la reproducción del evento en la aplicación propuesta.

La figura 13 muestra la visualización de la falla trifásica sólida simulada vista por el relevador de distancia, se incluye el efecto del filtro analógico y la ventana de muestreo de un ciclo (figura 13a) y dos ciclos (figura 13b). El efecto del tamaño de la ventana se puede cuantificar en el tiempo de operación, siendo mayor en la ventana de dos ciclos. La falla simulada fue sin resistencia. Diferentes condiciones pueden ser consideradas en 
la herramienta de simulación para mostrar mediante un análisis visual el efecto de la resistencia de falla en la operación de la protección de distancia, así como el comportamiento con diferentes tipos de características.

La simulación de oscilaciones de potencia y su efecto en la operación de la protección de distancia puede apreciarse en la figura 14.

(a)

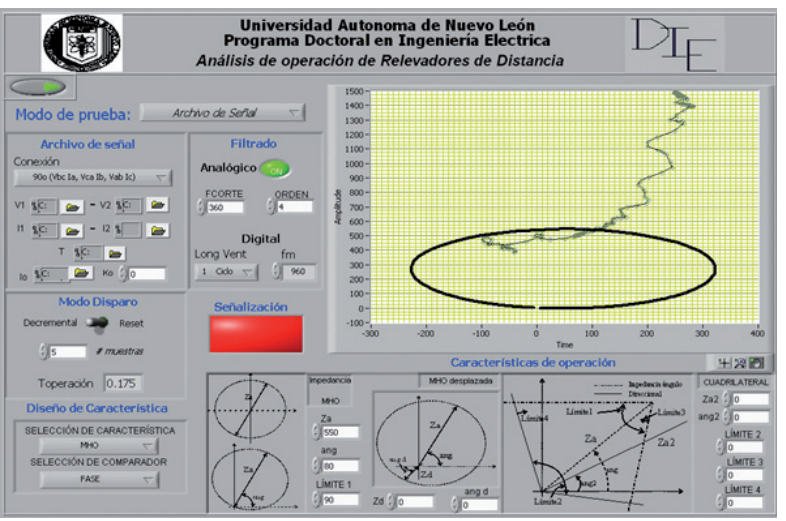

(b)

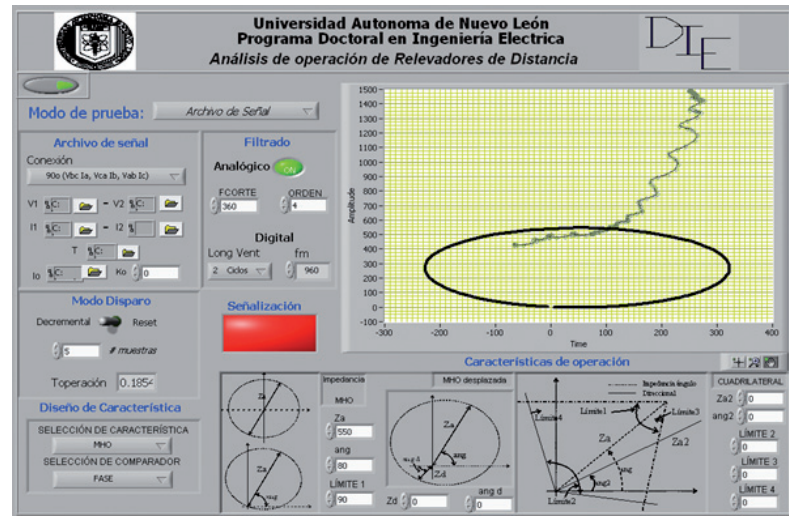

Figura 13. Simulación de una falla en PSCAD y visualización en la herramienta propuesta, a) ventana de datos de un ciclo, b) ventana de datos de dos ciclos

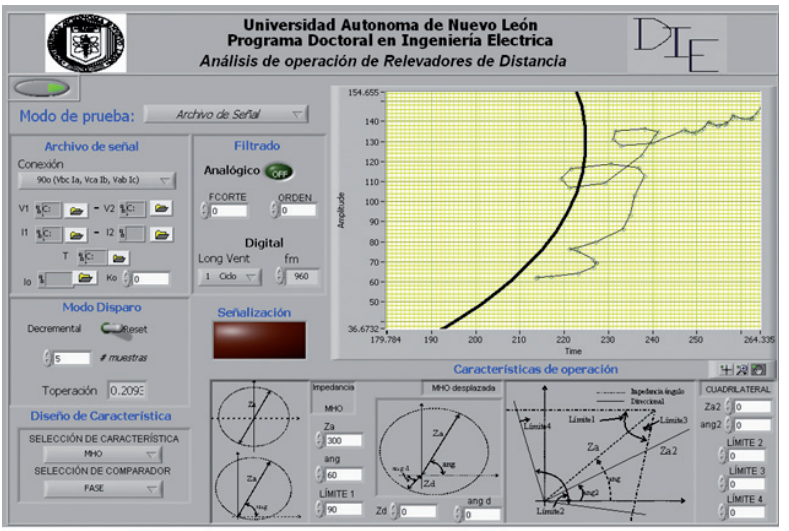

Figura 14. Simulación de una oscilación de potencia en PSCAD

\section{Conclusiones}

La herramienta de un relevador virtual propuesta en este trabajo ofrece alternativas didácticas que permiten el análisis del diseño de características de operación de relevadores de distancia y la evaluación funcional en estado dinámico ante diversas condiciones operativas, la interacción con diversos programas de simulación permite la exploración y análisis de un amplio espectro de eventos. Así, el efecto de diversos componentes del sistema tal como FACTS, aerogeneradores y limitadores de falla en el relevador de distancia pueden ser evaluados.

El modelado de un relevador de distancia en diversos programas comerciales de análisis resulta muchas veces limitado por la capacidad de estudio del mismo programa, la orientación de cada programa es diferente, así como las limitantes para la programación de algoritmos, por ejemplo, el desarrollo del algoritmo del relevador de distancia en EMTP resulta únicamente por el análisis del impacto de señales transitorias en la operación del relevador, otros escenarios y condiciones de operación no se pueden evaluar. Esta alternativa permite la aplicación de señales provenientes de diversas condiciones de operación sin necesidad de programar el modelado del relevador en diversas plataformas computacionales.

\section{Referencias}

Alvarado F.L., Lasseter R.H., Liu Y. An Integrated Engineering Simulation Environment, en: Proceedings of the 1987 IEEE PICA Conference, (1987, Montreal, Canadá), pp 213-221.

Alvarado F.L., Lasseter R.H., Kwon H., Mong S.K. A Module Oriented Emtp Interface. IEEE Transactions on Power Apparatus and Systems, 103(12):3488-3495, 1984.

Brahma S., De La Ree J., Gers J., Girgis A.A., Horowitz S., Hunt R., Kezunovic M., Madani V., McLaren P., Phadke A.G., Sachdev M.S., Sidhu T.S., Thorp J.S., Venkata S.S., Wiedman T. The Education and Training of Future Protection Engineers: Challenges, Opportunities and Solutions. IEEE Transactions on Power Delivery, 24(2):538-544, 2009.

Cook V. Analysis of Distance Protection, $1^{\text {a }}$ ed., Inglaterra, RSP Gales, John Wiley and Sons Inc, 1985.

Daneshdoost M., Shaat R. A Pc Based Integrated Software for Power System Education. IEEE Transactions on Power Systems, 4(3):1285-1292, 1989.

Hirsch P.M. Interactive Graphics for Load Flow, en: IEEE Power lndustry Computer Applications Conference Proceedings, (1977, Toronto, Canadá), pp 159- 165.

Huang J.A., Galiana F.D. An Integrated Personal Computer Graphics Environment for Power System Education, Analysis 
and Design. IEEE Transactions on Power Systems, 6(3):12791285, 1991.

Jian Y., Anderson M.D. Teaching Tool Shows Results Through Visualization [Power Systems Education]. Computer Applications in Power, IEEE, 11(1):37-42, 1998.

Jordan J.A., Schlaepfer F.M. An Interactive Computing Environment for Power System Planning, en: IEEE Power Industry Computer Applications Conference Proceedings, (1997, Toronto, Canadá), pp 166-174.

Lo C.H., Anderson M.D., Richards E.F. An Interactive Power System Analyzer with Graphics for Educational Use. IEEE Transactions on Power Systems, 1(2):174-181, 1986.

McLaren P.G., Mustaphi K., Benmouyal G., Chano S., Girgis A., Henville C., Kezunovic M., Kojovic L., Marttila R., Meisinger M., Michel G., Sachdev M.S., Skendzic V., Sidhu T.S., Tziouvaras D. Software Models for Relays. IEEE Transactions on Power Delivery, 16(2):238-245, 2001.

National Instruments (1997). Labview User Guide Version 7.0, Austin, National Instruments Corporation,

Gerhard-Ziegler. Numerical Distance Protection Principles and Applications, Siemens AG, Berlín y Munich, 1999.

Papadopoulos M., Hatziarfyriou N.D., Papadakis M.E. Graphics Aided Interactive Analysis of Radial Distribution Networks. IEEE Transactions on Power Delivery, 2(4):1297-1302, 1987.

Peterson J.N., Wall R.W. Interactive Relay Controlled Power System Modeling. P. IEEE Transactions on Power Delivery, 6(1):96102, 1991.

Proakis J.G., Manolakis D.G. Tratamiento digital de señales, Madrid, Prentice Hall, 1998.
Salon S.H. An Interactive Computer Graphics Package for Electric Power Engineering Education. IEEE Transactions on Power Apparatus and Systems, 102 (7):1954-1959, 1983.

Standard Requirements for Instrumentals Transformers. IEEE Standard, C57.13-1993, 1993.

Tziouvaras D.A., McLaren P., Alexander G., Dawson D., Esztergalyos J., Fromen C., Glinkowski M., Hasenwinkle I., Kezunovic M., Kojovic L., Kotheimer B., Kuffel R., Nordstrom J., Zocholl S. Mathematical Models for Current, Voltage, and Coupling Capacitor Voltage Transformers. IEEE Transactions on Power Delivery, 15(1):62-72, 2000.

Wachal R.W., Tarnawecky M.Z., Swift G.W. A Power System Analysis Package for Students, Using Computer Graphics. IEEE Transactions on Power Apparatus and Systems, 103(2):445448, 1984.

Warrington A.R., Van C. Protective Relays their Theory and Practice Volume Two, England, Chapman and Hall, Londres,1969.

Wei-Jen L., Jyh-Cherng G., Ren-Jun L., Didsayabutra P. A Physical Laboratory for Protective Relay Education. IEEE Transactions on Education, 45(2):182-186, 2002.

Westinghouse Electric Corporation. Applied Protective Relaying- $A$ Silent Sentinels Publication, Newark, N.J. Relay-Instrument Division, 1976.

Yu D.C., Chen S.T., Bischke R.F. A Pc Oriented Interactive and Graphical Simulation Package for Power System Study. IEEE Transactions on Power Systems, 4(1):353-360, 1989.

\section{Semblanza de los autores}

Luis Alonso Trujillo-Guajardo. Se graduó como ingeniero mecánico electricista en la Universidad Autónoma de Nuevo León (UANL) en 2005, y obtuvo el grado de maestro en ciencias de la ingeniería eléctrica con orientación en sistemas eléctricos de potencia en agosto de 2008 por la Facultad de Ingeniería Mecánica y Eléctrica (FIME). Actualmente es estudiante de doctorado en ingeniería eléctrica en el Programa Doctoral en Ingeniería Eléctrica en la FIME de la UANL donde también labora como profesor. Sus áreas de interés son el diseño y coordinación de protecciones de sistemas eléctricos de potencia, así como la operación y control de centrales de generación eólica y fuentes alternas de generación de energía eléctrica.

Arturo Conde-Enríquez. Se graduó como ingeniero mecánico electricista en la Universidad Veracruzana en 1993. Obtuvo la maestría en ciencias de la ingeniería eléctrica y el grado de doctor en ingeniería eléctrica en la Universidad Autónoma de Nuevo León en 1996 y 2002, respectivamente. Actualmente es profesor investigador del programa doctoral de ingeniería eléctrica de la FIME-UANL y es miembro del sistema nacional de investigadores. 